\title{
Study on Intelligent Socket Based on Hybrid Dynamic Systems
}

\author{
Zong-Feng SONG \\ Electronic Department, \\ Guangzhou College of Technology and Business, \\ Guangzhou, China \\ szfdyx@126.com
}

\begin{abstract}
Electrical equipment connecting socket outlets still consumes energy when in standby mode or shutdown state. This makes a large amount of power wasted. According to usage and operation characteristic, socket with multiple outlets can be modeled as hybrid dynamic systems. This paper proposes the basic architecture of intelligent socket, abstracts two-tuple model to memorize states, constructs automata model of socket hybrid dynamic systems, and a control algorithm is designed based on the automata model. In order to reduce the number of states to memorize, the actual current of socket is standardized. The operation example of intelligent socket is described and its future development is discussed. This intelligent socket can produce new states of electrical equipment through human-machine interaction and memorize them in its non-volatile memory. With the number of use increasing, the socket stores more and more states and become more and more intelligent, and can cut off power automatically when appliances stop running and save a lot of power.
\end{abstract}

Keywords--Intelligent Socket, Hybrid Dynamic Systems, Energy Saving and Emission Reduction, Embedded Systems.

\section{INTRODUCTION}

Due to the rapid economic and social development, power generation is dominated by thermal power during the past decades, which resulted in rapid growth of $\mathrm{CO} 2$ emission. Suffering from the two severe problems of energy crisis and global warming, energy saving and emission reduction must be implemented. It is a systematic engineering, which not only depends on the transformation of energy production modes and new power saving equipment, but also on the intelligent management of power utilization. For the reduction of $\mathrm{CO} 2$ emission, power generation mixes should be optimized by installing more new energy production equipment in the future. In order to realize the massive development and utilization of new energy, it is of great significance to promote development of large new energy bases such as hydropower, wind power, solar power, nuclear power etc. Many countries have been developing new energy and place more and more emphasis on them. By the end of 2011, the installed capacity of thermal power, hydropower, nuclear power, wind power is respectively $72.5 \%, 21.8 \%, 1.19 \%$ and $4.27 \%$ in China and the electricity production is respectively $82.54 \%, 14.03 \%, 1.88 \%$ and $1.54 \%$. In recent years, China has made a significant progress in the exploitation and use of new energy resources. During the year 2011, 371.2 billion RMB has been invested for national power engineering construction, $71.61 \%$ of which is for non-fossil fuel generation investment [1]. By the end of 2012, more than 20 percent of China's electricity came from the new energy and renewable energy [2]. Denmark saw renewable energy

This work was financially supported by Creative youth Foundation of Guangdong Province (2014KQNCX237). accounting for $23.6 \%$ of energy consumption in 2011. At the same time, electricity production based on renewable energy accounted for $40.7 \%$ of domestic electricity produced in the country [3]. The electricity from renewable energy accounted for nearly $22 \%$ of the total amount in Germany and about $31 \%$ in America in 2012[4,5]. The use of renewable energy has many potential benefits, including the reduction in greenhouse gas emission, the diversification of energy supplies and the reduced dependency on fossil fuel markets. So the development and utilization of new energy should be enforced.

For saving energy, new power-saving equipments have been developed through optimizing devices' structures and adopting energy saving technology. One of which is variable frequency technology. Many types of equipment, designed nowadays such as heating, ventilation air conditioning devices and so on, can consume different power volume when working through variable frequency technology. As we know, when current flow is controlled without regulating the motor speed, it runs continuously at full speed. This makes significant energy wasted over most of its operating time. However variable frequency technology can control motor's speed according to environment, so the device can greatly reduce the power consumption and the energy saving effect is in general more than $10 \%$. Except obvious benefit in energy saving, it can also bring environmental protection, improve the energy utilization rate, prolong service life of equipment, reduce operating cost and maintenance cost of equipment and ensure electricity quality and reliability of power for users. This technology has been used in a wide range of applications.

Even the embedded and real-time systems can also use dynamic voltage scaling (DVS) technique to reduce processor's energy consumption through changing the processor's supply voltage and clock frequency at the runtime, which is one of the most effective techniques for reducing energy consumption [6].

Because of circuit design and electrical characteristics, most of equipment still consumes power in standby mode and shutdown state. The total of power waste is very large. And this also makes equipment reduce their life or even gotten damaged when power quality changes. According to materials provided by Shanghai energy conservation association [7]: A ordinary outlet's power in no load is about $1.9 \mathrm{~W}$; the standby power consumption of a host is about $4.81 \mathrm{~W}$ after power is off; the standby power consumption of a display is about $30 \mathrm{~W}$; and the standby power consumption of a printer is about $12 \mathrm{~W}$; and other appliances in standby mode can also consume much energy. In America, power utilization in home and offices accounts for about 76 percent of the total electric energy consumption [8]. And they are the very places where most of power waste of 
shutdown state and standby mode happens. The power waste of standby state in America reaches about 52TW every year. According to researches, the standby power waste accounts for $3 \% \sim 13 \%$ in national civil power in OECD (Organization for Economic Cooperation and Development)[9]. It is estimated that there could be hundreds of millions of computers, televisions and monitors in China, the whole power waste could be trillions of $\mathrm{KWH}$. With the environment of energy saving and emission reduction occurring, the standby power waste of electrical equipment should not be overlooked.

There is a kind of IC card widely used in hotel, which can cut off whole power of room when people leaving the room extract it from slot and power on when it is inserted into slot. This can save some power for hotels. This card can be promoted in other suitable places. However, it does not apply some places where there is equipment which needs a continuous power supply. So this energy saving method has a certain limitation. Intelligent sockets, which have abilities to monitor working states of electrical equipment and to decide whether to cut off the power by itself, are more flexible than the above IC card in power energy management and have wider applications. This kind of socket can make great contribution to energy saving and emission reduction.

Electrical socket is the most common power connection point in home, offices and other places. There have been some energy saving sockets. Wenxuan Yao has designed a multi-functional intelligent socket using low-power SCM PIC16F877A for data processing and controlling. It has functions like temperature detection, over-current/voltage protection, automatic alarming, and automatic dormancy [10]. However, the upper/lower limit values of parameters, like temperature, voltage, current and so on, should be pre-set. It is difficult for people to memorize the upper/lower values of parameters in practice use. So it's not flexible enough, especially when it comes to estimate whether to shut off power.

The intelligent socket designed by Kai Zhang using Upsd32xx Soc SCM as the control core has functions to set, allocate and limit load power. It could satisfy the power for homes and limit the high power load in power peak hours [9]. Although cutting off power when equipments staying in the standby mode, the socket has low intelligence due to needing to set up a fixed value previously at which it switches off power. It can't successfully cut off power when the actual voltage deviates from the standard one.

The authors of papers $[11,12]$ developed a socket with sensors, which can collect power consumption data and can switch on or off the power supply to plugged-in appliances. Each of power socket sensors is individually identified by actuator, which can send a command stream to control the switching off/on of power socket sensors. But the socket can't judge the standby mode and cut off power by itself.

Paper [13] describes an output adapter which has a checker. It can check standby mode and shutdown state of appliance and can switch on/off power source according to them. The adapter can be installed in socket. But every extension of socket needs one adapter, which makes the socket more expensive. Moreover this kind of adapter is now just prototype and not tested yet.
The authors of paper [14] presented a smart plug named nPlug that sits between the wall sockets and deferrable loads such as water heaters, washing machines, and electric vehicles. The nPlug combines real-time sensing and analytics to infer peak periods as well as supply-demand imbalance and reschedule attached appliances in a decentralized manner to alleviate peaks whenever possible. They do not require any manual intervention by end consumer or any enhancement to appliance or existing infrastructure. Experiments indicate that the nPlug can be an effective and inexpensive technology to address peaking shortage.

The authors of paper [15] developed a smart plug with a wireless sensor network for monitoring electrical usage in smart house. The smart plug integrates an AC power socket, a relay to switch the socket on/off, a current transformer sensor and a Kmote. The Kmote is a wireless communication interface based on TinyOS. The socket integrates AC power receptacle and wireless sensor node to switch power on/off and to measure the power consumption of plugged appliances. But it emphasizes on power consumption and hardly mentions power on/off according to equipment operation state.

Paper [16] introduces a low cost solution to power on/off home appliances using a new power distribution system. In this system, appliances do not need to have their own communication/control units. An RFID tag is built in the plug of each appliance, which ID is read by the RFID reader in the connected socket, and then it is sent to smart meter even through power strips and/or extension cords. This proposal has three features: (1) the smart meter identifies appliances connected to individual wall socket simply by connecting appliances to socket, and then enables itself to have their on/off control. (2) Additional cost to individual appliances is only that of a single RFID tag built in plugs, and the manufacturers do not need to redesign its body. So this system is thus appropriate for existing appliances. (3)This system also provides safety features for avoiding octopus wiring and detecting recalled appliances through avoiding different priority products into the same socket, totalizing the consumption power of all the connected appliances, and changing the connections if the overload is expected, and maintaining a list of recalled appliances via internet by identifying the recalled appliances the moment they are plugged in by smart meter. In this system, on/off controls of the appliances are made by wall sockets. But it is the smart meter not the socket itself that identifies appliances connected to individual socket and this system emphasizes on adjusting appliances using power at peak times.

Paper [17] introduces design and application of intelligent electrical outlet, which can measure effectively electricity data, control power supply line on/off, cut off electrical appliance's power at nonessential time interval, and thus achieves the goal of electricity saving and emission reduction. The outlet contains microprocessor, power signal measurement module, power conversion module, power control module, communication module, and display and alarm module. The main program of intelligent electrical outlet mainly includes monitoring and processing measurement signal module, communicating module and controlling power on/off module, etc. When monitored data exceed limit value thresholds, MCU issues a warning signal and 
sends data to gateway controller by communicating module. The receiving part of communicating module uses interrupt mode. It rapidly responds the order from gateway controller, and controls relay on/off through interface. So the power of electrical equipment is shut off at unnecessary work intervals. There is a RFID tag in plug and a newly-designed sockets/power strips in this system. Experiments show that the intelligent electrical outlet can save electricity energy $10 \% \sim 40 \%$ or so. Along with electrical equipment components getting aging and standby energy consumption increasing, electricity saving rate will be further improved by using the intelligent electrical outlet. Because every outlet has a RFID reader which reads IDs of appliances, this socket is more expensive.

There are many other kinds of sockets which are used by some special appliances such as computer, TV Set, refrigerator and so on. They have simple functions of cutting off power sources and checking operation states of appliances, which save much energy and can contribute to constructing economical society. But they are so simple that they are only suitable for some special appliances. This limits their application.

Currently, one socket often supplies several appliances with or without correlations between each other. That means socket can be used by uncertain appliances freely. When all the appliances are in standby mode or shutdown states, they still consume a small amount of electrical energy. There are a large amount of appliances which use energy just as above.

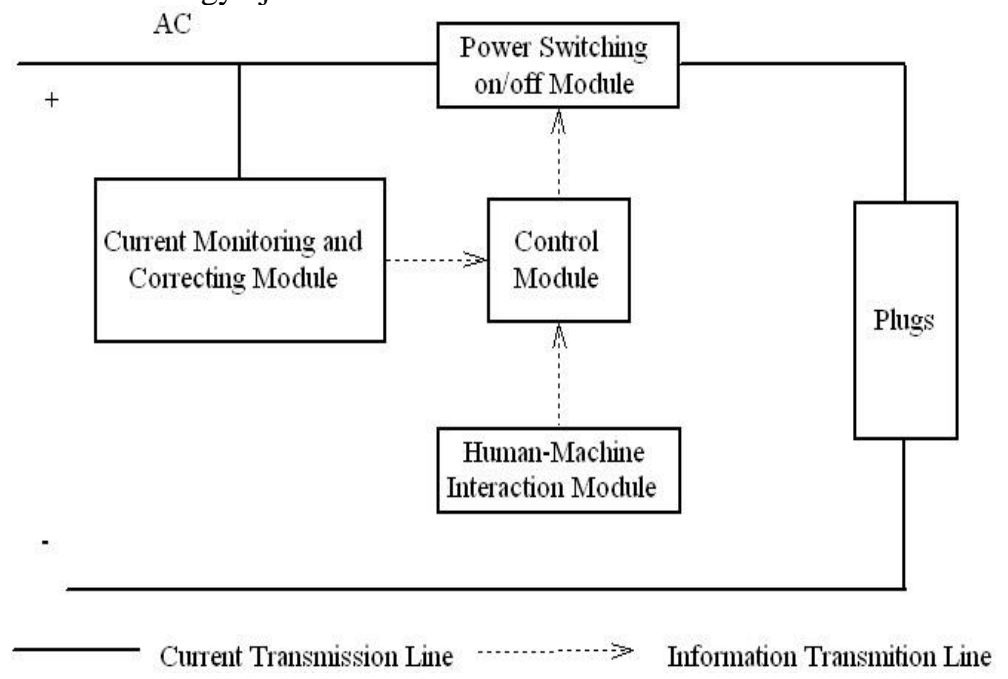

Fig. 1.Socket architecture
There is a standard voltage value in every power-using environment. But the actual voltage often fluctuates around the standard voltage because of the complex power-using environment. This produces many current values, and makes corresponding working states. But these different working states actually correspond to the same state of appliances. So in order to reduce the number of working states to be stored, the actual voltage value should be transformed into standard one. The voltage sensor monitors the voltage, and the current-correcting module revises the checked current value according to the actual voltage and standard one. The correcting from actual current to standard one makes the new working state, which is memorized view of this phenomenon, it is necessary to design a kind of socket which can supply several appliances, identify the overall can cut off power source to the socket when in these modes automatically. At the same time, it can interact with people; memorize equipments' power using states. And with the number of interactions increasing, it stores more and more states, and becomes more and more intelligent. The kind of intelligent socket can solve the problem caused by bad power using habits.

\section{BASIC ARCHITECTURE OF INTELLIGENT SOCKET}

The intelligent socket consists of current monitoring and correcting module, power switching on/off module, control module, and human-machine interaction module. The basic architectural model is shown in Fig. 1.

The current monitoring and correcting module consists of current sensor, voltage sensor and current-correcting module. The current sensor monitors current value of socket. According to this value, the system can judge if all the appliances connecting the socket are all in standby mode. Only when all the appliances are in standby mode, would the socket cut off power supply. For saving cost and place of socket, there is only one current checking sensor in the whole socket which checks current value of socket, not one or two appliances' current value.
Consequently, a large amount of electrical energy is wasted. In power shutdown state or standby mode of all equipments and

in storage. So the number of states the system needs to store is reduced greatly. The working state has two members, Current and Switch. The member Current records current value of socket. The value of member Switch shows if the socket should be cut off or on at this current value. The values of Current and Switch make one working state.

The controlling module mainly consists of microcontroller and control algorithm. It is the core of the intelligent socket. It receives the checked current value. If there is a state of which the Current's value is equal to the checked current value, it sends switching instructions to the power switching module according to the value of the state's Switch. Or it generates and stores a 
new state through the interaction with human. The human-machine interface presents an interface for human intervention through which the socket can become more and more intelligent. The power switching module mainly consists of a relay. It receives the switching instructions from controlling module and switches on or off power of socket.

\section{CONTROL OF INTELLIGENT SOCKET}

\section{A. State Model of Intelligent Socket}

Considering the using habits of electrical socket, the intelligent socket should have two working patterns, power on and power off. They are subjected to the working states of appliances which can be represented by the current values of appliances which connect to the socket. When all the appliances are in standby mode, the working pattern should be power off, or the working pattern should be on. So the working states of the intelligent socket can be represented by the total current value of the socket and its working pattern. So it can be abstracted as two tuple, which is showed as follows:

\section{State (Current, Switch)}

The member Current can get integer value which can smooth the current error caused by the grid voltage deviation from the standard one. The member Switch gets Boolean value, 1 standing for power on, and 0 for power off.

When the current monitoring module detects the current value of main power transmission line at a certain time interval, the control module compares it with the values of stored states. If the value of one state's Current is equal to this detected current value, power on or off instruction is produced according to the value of the state's Switch and it is sent to power switching on/off module. If there is no related state, a new state is produced according to the current value and the information from human-machine interaction module and stored in memory.

The intelligent socket is applicable to a wide variety of equipments, not limited to certain equipments. In order to reduce cost, it has only one current monitoring module which only detects the current of socket's main power supply line, not every single appliance's consuming current.

At the beginning, the intelligent socket has no states. With the number of utilization and interaction with human increasing, more and more states are produced and stored. So the socket becomes more and more intelligent. These working states are stored in non-volatile storage. They can't be lost when the socket loses power.

\section{B. Rectification of Current Value}

The deviation of actual voltage from the standard one will produce many different current values. However, these different current values maybe represents the same working state of a group of appliances. In order to save storage memory and indentify appliances' working states continently, the current value produced by actual voltage should be converted into standardized one corresponding to standard voltage.

If the actual voltage value is V1, the standard voltage value is $\mathrm{V}$, and the actual monitored current value is I1, the current value
I stored in the working state should be calculated by Equation (1).

$$
\mathrm{I}=\mathrm{I} 1 \times \mathrm{V} / \mathrm{V} 1
$$

Through this conversion, the socket stores less working states and improves the socket's performance.

\section{Control Model of Intelligent Socket}

Hybrid systems contain two distinct types of components, subsystems with continuous dynamics and subsystems with discrete dynamics. These systems typically contain variables or signals that take values from a continuous set and also variables that take values from a discrete, typically finite set. These continuous or discrete variables depend on independent variables such as time, which may also be continuous or discrete; some of the variables may also be discrete event driven in an asynchronous manner.

Hybrid systems are central in the analysis and design of intelligent control systems with high degrees of autonomy as they arise from the interaction of discrete planning algorithms and continuous control algorithms. From a modeling point of view, it is perhaps more realistic to model a phenomenon by a dynamic system that incorporates both continuous and discrete components.

According to the operation characteristics, the control system of intelligent socket can be modeled as a hybrid system. And it can be described as state automata. The basic component of automata is state. Each state has four parameters, namely, S, I, Ig and $\Delta \mathrm{t}$. Where $\mathrm{S}$ is state identification parameter, it has two kinds of value, OFF and ON. I is state maintenance parameter. In every state, I has a solid value. In this hybrid system, I can be modeled as follows:

$$
\mathrm{I}=\mathrm{f}(\mathrm{R}, \mathrm{V} 1, \mathrm{~V})
$$

I is the total current of the socket, $\mathrm{R}$ is the total resistance, $\mathrm{V} 1$ is the actual voltage, and $\mathrm{V}$ is the standard voltage. In the practical use, the value of I is gained by current sensor directly.

The parameter $\Delta \mathrm{t}$ is state transformation condition. It records the lasting time of one stable current value. If one current value changes in the time of $\Delta \mathrm{t}$, the value of $\Delta \mathrm{t}$ should be recalculated. When working in one ON state, if the checked current is not equal to I's value and the value lasts over the time of $\Delta \mathrm{t}$, then the socket transforms to another state. When staying in OFF state over the time of $\Delta \mathrm{t}$, the socket will power off. If the checked current is not equal to the I's value of the OFF state and the value lasts over the time of $\Delta t$, the socket transforms to another state.

In order to record the changing current value timely and calculate the value of $\Delta \mathrm{t}$ conveniently, a global variable Ig is set which records the sampled current value. When the socket is powered on, it can sample a current value and work in a corresponding ON state of which I's value is equal to the sampled current value. If there is no corresponding ON state, the system produces a new $\mathrm{ON}$ state according to the current value. When the socket is working in one ON state, the control system samples at certain period continually and stores the value in the Ig. When finding a stable value of Ig which is equal to the value of one state's I, the system enters this state after $\Delta t$ and power on or off according to the value of S. If there is no corresponding 
state and the value of Ig is bigger than the value of I, the system produces a new state. In this state, I's value is Ig's value and S's value is ON. If the value of Ig is less than the value of I, the system gets S's value through human-machine interaction and produces a new state. The use of Ig makes the controller unnecessary to work when the socket is powered off. This simplifies socket's architecture, reduces energy consumption and development cost.

According to the above description, the automata model can be made for the control system just as shown in Fig. 2. In this model, the ON or OFF state does not represent a certain specified state, but has universal significance.

The automaton is dynamic. At the beginning, there are no states. With the number of human-machine interaction increasing, the automaton extends, and the socket becomes more and more intelligent. The tstart is the time when a new current value occurs, and the tend is the time when this current value ends. If the socket is working in one ON state and its value of I is not equal to the value of $\mathrm{Ig}$, and the value of $\Delta \mathrm{t}$ is bigger than the corresponding set value, the socket would transform to another state.

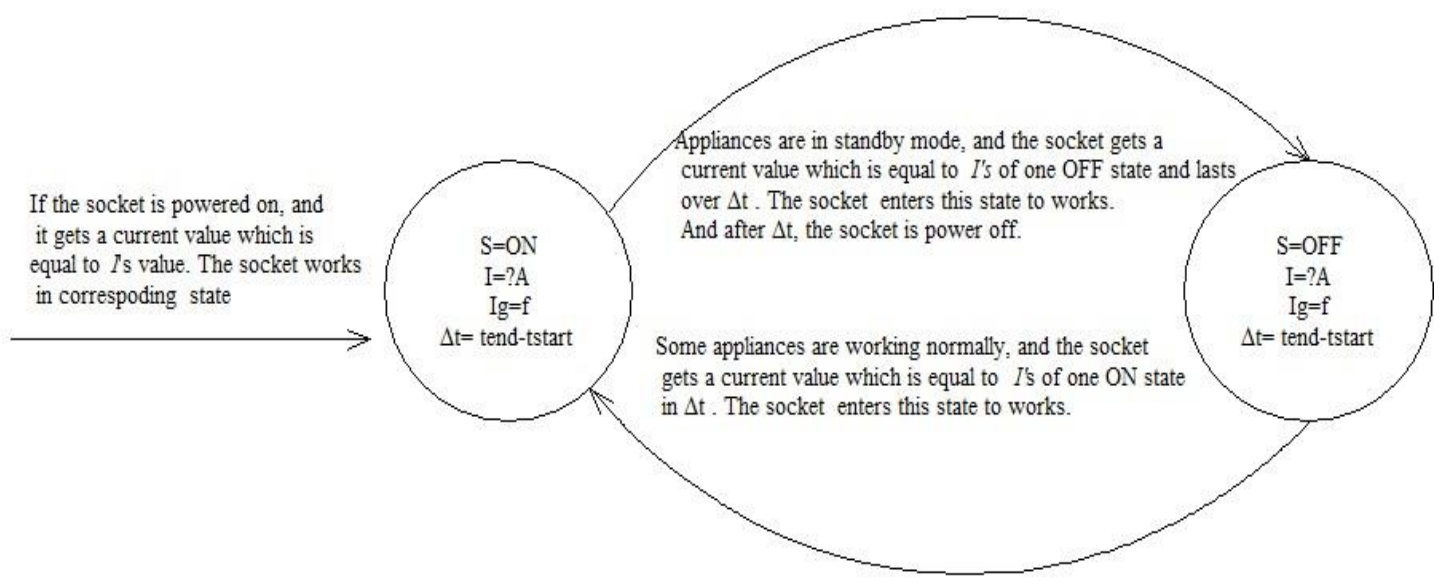

Fig. 2.Automata model of socket control system.

\section{CONTROL ALGORITHM OF INTELLIGENT SOCKET}

When the socket is used for the first time, there is no state in control module. When the socket is powered on via the human-machine interface, current monitoring and correcting module gets a current value which is set as Ig's value. The socket enters corresponding state to work according to Ig's value. If there is no state of which I's value is equal to Ig's value, a new state is produced and added into automata. Then the socket works in this state. Whenever the current changes, its value is set into Ig. When the value lasts longer than $\Delta t$, the socket enters a corresponding state to work or power off. If there is no corresponding state and the current value is bigger than that of I of the state, a new state occurs, of which I's value is the rectified current value by formula (1) and the member $\mathrm{S}$ is set as the value of 1 and the socket works in this state. If the current decreases, the new state's member $\mathrm{S}$ is set as the value of 0 , and the socket switches off. Then if the socket is switched on again via the human-machine interaction module, the $\mathrm{S}$ of the state above should be set as the value of 1 . With the number of utilization and human-machine interaction increasing, automata get more and more states, and the socket becomes more and more intelligent. The algorithm flow is shown in Fig. 3.

\section{EXTENDED FUNCTIONS}

The socket discussed above has only basic functions such as new state production, powering off/on according to the automata, and so on. However, more and more appliances nowadays have time-setting function. It is very convenient for people to control appliances remotely. For example, if someone gets off from work at 17:00, and wants to eat at 18:30, he can set cooker to work at 17:30 on his way home. In order to save energy, when the cooking is over, the socket should send signal to the person. Then the person could be able to power on the cooker remotely. This can be achieved easily with wireless communication technology[12,15]. 


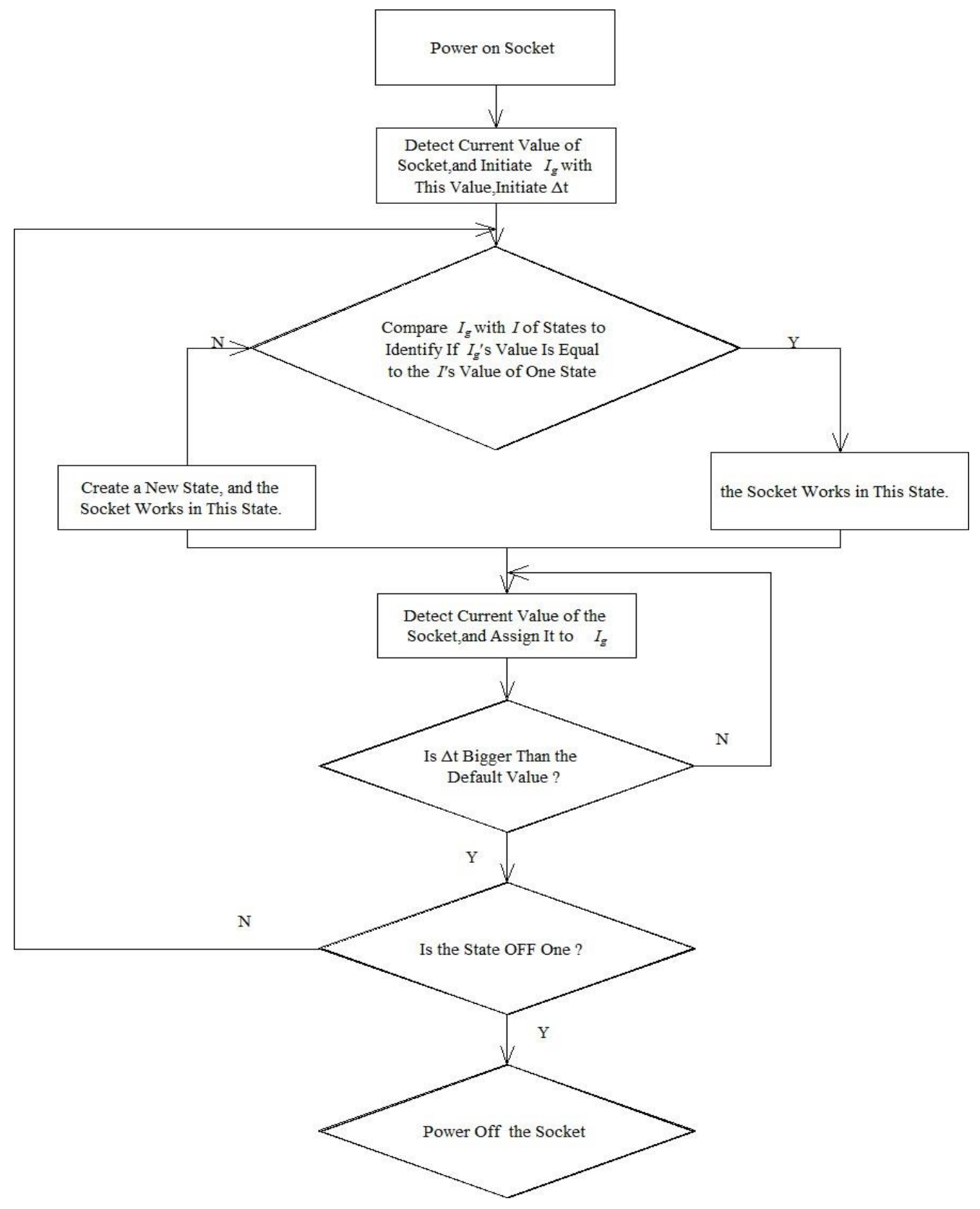

Fig. 3. Control algorithm flow 


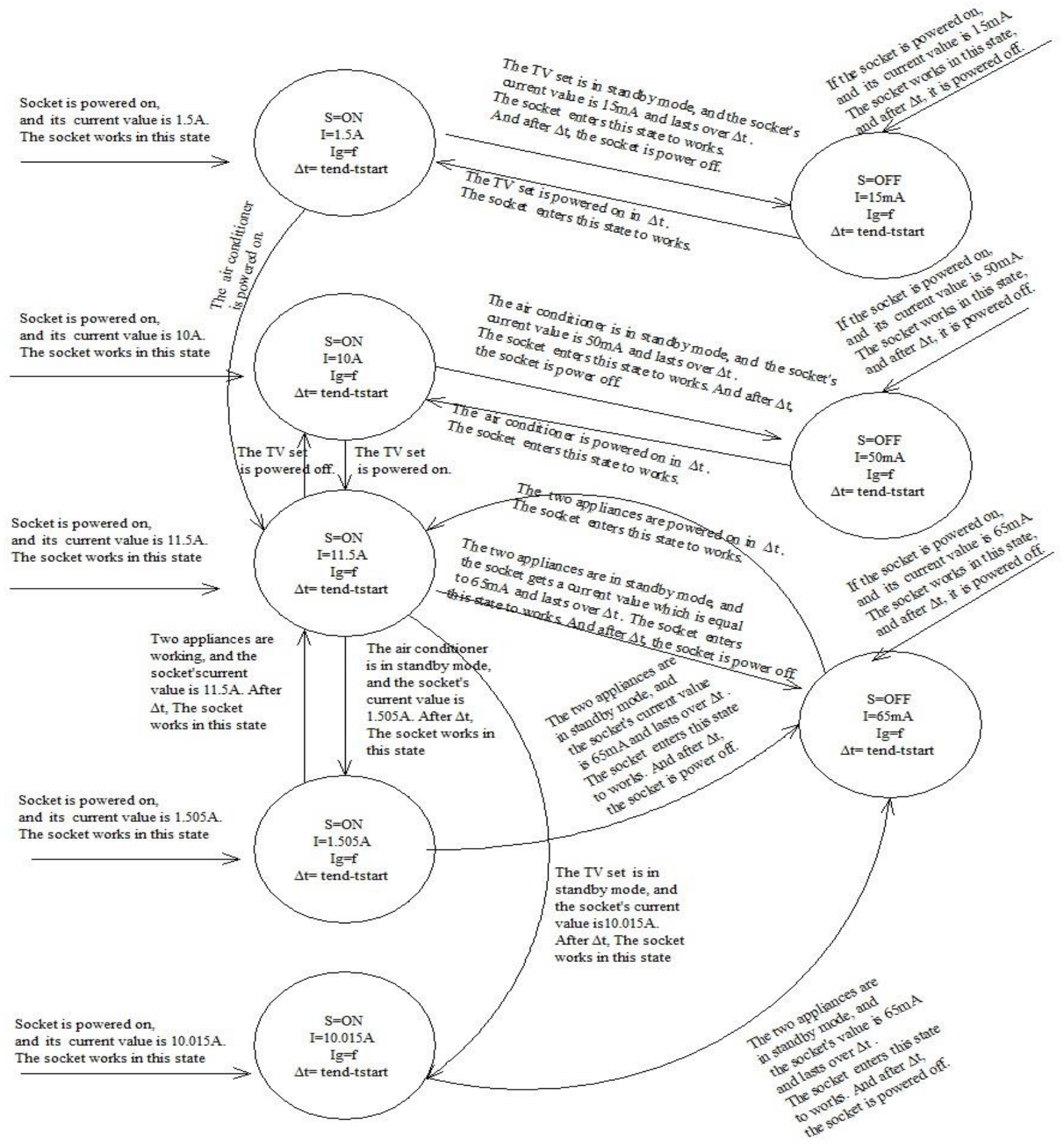

Fig. 4.Example of automata

\section{CONCLUSIONS}

Energy saving can be obtained from multiple perspectives. This paper focuses on the phenomenon that the appliances in the standby or shutdown mode still consume power and the poor energy using habits. For example, power is not cut off after equipment stops running. Based on these phenomena, an intelligent socket is proposed with the abilities to check the operation state of appliances and switch off its own power supply automatically. With the number of appliances in use and human-machine interaction increasing, the socket owns more and more states. According to these states, the socket can supply appliances with the needed power, and cut off the power supply when appliances are in the standby mode or in the shutdown state. It can be a good helper for people who do not cut off power supply after using equipment and can save a large amount of power. Moreover, because of the utilization of microcontroller, the socket can easily have other functions to interact with other independent devices or systems for power on or off, peak and valley power allocation, safe electricity utilization.

\section{REFERENCES}

[1] ]M. Zeng, S. Xue, M.J. Ma and X.L. Zhu. "New energy bases and sustainable development in China: A review", Renewable and Sustainable Energy Reviews, Vol. 20, pp.169-185, 2013.

[2] Information on http://www.cmsino.com/zixun_liebiao_ny_2463_2.html.

[3] Information on

http://cleantechnica.com/2012/09/28/danish-renewable-energy-generatio n-wind-energy-generation-percentage. 
[4] Information on http://www.askci.com/news/201301/24/ 241352457246.shtml.

[5] Information on http://www.eia.gov/tools/faqs/faq.cfm? id=427\&t=3.

[6] F. Xia, L.H. Ma, W.H. Zhao, Y.X. Sun, J.X Dong, "Enhanced Energy-Aware Feedback Scheduling of Embedded Control Systems", JOURNAL OF COMPUTERS, VOL. 4, NO. 2, pp.103-111, February 2009.

[7] P.J. Xu, "New technologies of communication auxiliary equipment energy saving", the collection of China communication networking committee conference in 2009 (final volume). Xi'an, 2009, pp. 1160 1164.

[8] Information on http://buildingsdatabook.eere.energy.gov.

[9] K. Zhang, Q.X. Dan and F.B. Weng, "Design and implementation of the new digital energy-saving power distribution intelligent socket", Electronic Design \& Application World-Nikkei Electronics China, Vol. 7 , pp.90-93, 2009.

[10] W.X. Yao, Z.S. Teng and J.W. Xiong, "The Design of Multi-Functional Intelligent Socket", ECHNOLOGICAL DEVELOPMENT OF ENTERPRISE, Vol .29, No. 11pp.28-30, 2010,.

[11] S. Veleva, M. Kacarska and D. Davcev, "Box-Dimension as a Correlation Measure for Data Mining of Power Socket Sensor Data", in IEEE International Conference on Smart Measurements for Grids, 2011, pp.88-94.
[12] J. Yun, S.S. Lee and I.Y. Ahn, M.H. Song, M.W. Ryu, "Monitoring and Control of Energy Consumption Using smart Sockets and Smart phones", in Computer Applications for Security, Control and System Engineering, 2012, Vol.339, pp284-290 .

[13] A.S. Kulkarni, K.C. Welch and C.K. Harnett, "A review of electricity monitoring and feedback systems", in IEEE Southeast Con 2011 Building Global Engineers, 2011, pp.321-326.

[14] T. Ganu, D.P. Seetharam and V. Arya, R. Kunnath, J. Hazra, et al., "nPlug A smart plug for alleviating peak loads", in the 3rd International Conference on Future Energy Systems: "Where Energy, Computing and Communication Meet", 2012.

[15] M.G. Lee, Y.K. Park, K.K. Jung, J.J. Yoo, "Wireless Electricity Monitoring System for Smart House using Smart Plug", in The 15th World Multi-Conference on Systemics, Cybernetics and Informatics, Vol.2, 2011, pp. 16-19.

[16] T. Noma, Y. Ookuma, "Power and Safety Management of Electrical Appliances in a Smart Power Distribution System", in 2011 IEEE International Conference on Consumer Electronics (ICCE), 2011, pp. 511-512.

[17] Y. Ma, "The Design and Application of Intelligent Electrical Outlet for Campus's Electricity Saving and Emission Reduction", JOURNAL OF COMPUTERS, Vol. 7, No. 7, pp.1696-1703, July 2012. 